\title{
OBSERVATIONS OF THE GALACTIC HALO
}

\author{
Klaas s. de Boer \\ Sternwarte, Universty of Bonn, F.R. Germany
}

\begin{abstract}
Summary. After reviewing the history of the early research on gas in the Milky way halo, observational methods to investigate the halo are described. The meaning of the concepts Halo and HVC is discussed and selectional bias in databases is pointed out. The essentials of the interpretation of observed velocities are given. The scanty information on distances of HVCs is reviewed and an outlook is given on the possibilities to firmly settle the distance of at least a few HVCs.
\end{abstract}

\section{General History}

The concept of a halo around the disk of the Milky Way dates from the studies of stellar distributions at the beginning of the century. The globular stars clusters appeared to exist in a roughly spherical volume around the disk, a space called halo. spectroscopy then showed that stars at high galactic latitudes have, on average, a lower metal content than stars in the solar vicinity. The nature of these stars tends towards the properties of the globular cluster stars and Baade named these field objects stars of the halopopulation. The astronomical use of the word halo was taken from the meaning "halos" of saints, but it originally derives from the greek word for "(round) threshing ground".

The threshing ground aspect of the word halo remained meaningless to me until three years ago. During an observing stay at the Calar Alto observatory looking for halo gas, I spent (after a misty night) a pleasant afternoon with my colleagues touring the valleys of the Sierra de los filabres. At a very scenic spot we noticed an abandoned village and decided to take a look. At an elevated part, away from the few crumbling walls of foregone houses, we found such a "halos", a circular well-paved area. I imagined how the people of the hamlet would have threshed their grains using the convective airflow up into their valley and past their village to produce clouds of small and light particulate stuff high into the air.

The studies of the interstellar gas in the late 1930's had revealed that many a line of sight showed multiplicity in the interstellar absorption spectra. Adams (1949) reports 2 stars at b $>20^{\circ}$ having multiplicity in CaII $k$. To follow up on these findings Guido Münch started to systematically observe stars at high galactic latitude (Münch 1952). The full data appeared much later (Münch and zirin 1961). Several absorption structures were found with velocities substantially different from the expected $0 \mathrm{~km} \mathrm{~s}^{-1}$ for local galactic gas. These structures then represented High Velocity 
Clouds (HVCs), at that time defined as clouds with velocities differing by more than $20 \mathrm{~km} \mathrm{~s}^{-1}$ from the LSR. Based on the distances of the stars used as background light sources we know roughly how far from the Milky Way disk the clouds detected by Münch in the visual are.

The radio $21-\mathrm{cm}$ measurements at high galactic latitudes showed the presence of $\mathrm{HI}$ gas with surprisingly large velocities. Isolated features were found, but also larger complexes were discovered, like Complex $C$ in the northern sky. Most clouds seemed the move towards the Solar Neigbourhood (see review by van Woerden et al., 1985). For none of these structures detected at $21 \mathrm{~cm}$ could distances be derived; the presence of these clouds could not be confirmed through absorption-line measurements in stellar spectra. This by itself implied rather large distances from the Milky Way disk. Later systematic surveys in both the northern and southern hemisphere (Giovanelli 1980; Hulsbosch 1985; Bajaja et al 1985; Hulsbosch and Wakker 1988) demonstrated that about $1 / 3$ of all directions at high latitudes show the presence of HVCs.

The likely existence of large volumes of ionzed gas was most elegantly advocated from theoretical considerations by spitzer (1956). This proposition was based on the early results of the observations of Guido Münch, of which spitzer was informed during frequent visits from Princeton to the Mount Wilson Observatory. The very presence of neutral clouds had to mean that there was a confining medium as well, and since there was no observational evidence for other forms of cool or neutral gas, spitzer postulated that the space outside the disk was filled with hot and well ionised gas possibly out to $10 \mathrm{kpc}$ and with a temperature of the order of a million degrees.

In the following, methods to study halo gas are reviewed and results are described. A number of reviews has appeared on the topic of gas in the halo such as by York (1982), de Boer (1985), Bergeron, Savage, Green (1987).

Ultraviolet, X-ray, Infrared and H-alpha Data

With the opening of the UV-part of the spectrum in 1972 resonance transitions of highly ionised states of interstellar atoms finally (Spitzer 1956) became accessible. The first unambigous proof for the existence of well ionised gas outside the disk of the Milky Way came with the IUE from the high dispersion spectra of stars in the Large Magellanic Cloud (Savage and de Boer 1979, 1981). Absorption by CIV and SiIV was detected at velocities up to $140 \mathrm{~km} \mathrm{~s}^{-1}$ LSR, velocities almost certainly pertaining to gas of the Milky Way. This gas clearly is unaffected by the ionisation environment of the background light sources. Earlier studies on the possible existence of hot and ionised gas generally distributed in the Milky Way disk (see Jenkins 1978) always had to consider the possibility of ionisation by the radiation field of the actual star observed.

The measurements of the absorption lines in the ultraviolet have a 
definite advantage over those in the visual. In the UV one finds the transitions of the elements abundant in the gas phase in neutral clouds such as CII and MgII and also the numerous lines of SiII and FeII. These have on average an optical depth more than a factor 100 larger than the lines in the visual, thus making the detection of small amounts of gas, in particular at exceptional velocities, relatively easy. However, the UV instrumentation available up to now is attached to the rather small $45 \mathrm{~cm}$ IUE telescope. Also the gas-phase abundances of depleted elements (such as TiII) may be a function of distance from the galactic plane (Albert 1983; Edgar and Savage 1989).

The metal content of HVCs seen at high galactic latitudes is not too far from the solar composition. The very fact that absorption Iines from metals, such as from CaII, FeII, MgII, CII, etc., are seen (toward globular clusters and toward the LMC, see below) shows that HVCs consist largely of processed material. CaII has been found in HVCs using a number of extragalactic background light sources, but the $\mathrm{Ca}$ abundance is uncertain due to possible depletion effects. The abundances of FeII, MgII, etc., could be determined for the HVCs on the lines of sight to the LMC (Savage and de Boer 1981) to be within 5 and $25 \%$ of solar. Having recorded HI $21-\mathrm{cm}$ emission profiles showing these HVCs, McGee, Newton, and Morton (1983) and McGee and Newton (1986) could fix the metal content of the HVCs at about $40 \%$ of solar while the high signal-tonoise data toward SN1987A showed this to be the case for gas in three HVCs on that line of sight (Blades et al 1988). Suggestions that the -60 and $-130 \mathrm{~km} \mathrm{~s}^{-1}$ clouds are associated with the LMC are negated by observations by de Boer, Morras, and Bajaja (1989).

During the 1970 's, Wisconsin rocket soft $x$-ray scans of the almost complete sky showed that there is a fair amount of soft $x-r a y$ background intensity of which it was speculated that it might come in part from the Milky way halo (the full account of the data is given by McGammon et al 1983). The spatial structure in the background appears to be anti-correlated with the location of HI clouds as known from 21-cm measurements and the background flux reduces nowhere on the sky to zero level. The explanation is that there is a local source of soft $X$-ray emission (local hot bubble) and neutral clouds absorb the soft $x$-rays coming from a more distant source, thus producing "cloud-shadows". Recent reanalyses of the all-sky soft X-ray data (Hirth et al. 1989, snowden and Sanders, these proceedings, show that the observed intensities fit to a $10^{\prime} \mathrm{K}$ medium whose source function increases with $z$-distance of about half a $\mathrm{kpc}$ and in which neutral clouds are embedded.

The IRAS satellite revealed the widespread presence of thin veils of IR emitting dust at high galactic latitudes which was named infrared cirrus (Low et al 1984). Essentially all filaments have been identified with HI 21-cm gas, and known high latitude diffuse reflection nebulae coincide with IRAS cirrus as well. For one of these features the distance could be derived using the gas density distribution towards stars in almost the same directions as derived from the increase in strength of absorption lines (Hobbs, Blitz, Magnani 1986). Also studies using star counts (Magnani and de Vries 1986) and generalised surveys of the CO associated with the IRAS cirrus (Magnani, Lada, and Blitz 1986) led to distances < 200 pc. 
For most of the individual cirrus the distance is still unknown.

H-alpha emission from diffuse galactic gas has intensively been investigated by Reynolds since 1978 with a Fabry-Perot device allowing a velocity resolution of $12 \mathrm{~km} \mathrm{~s}^{-1}$. Among the multitude of directions and objects to look at also a few known HVCs have been measured. In those directions Reynolds (1987) sets firm upper limits to the intensity of $H$-alpha radiation. This relative lack of H-alpha emission indicates that the ionised gas surrounding the HVCs has a density $n(e)<0.1 \mathrm{~cm}^{-1}$ or a temperature $T>10: \mathrm{K}$, or both. Also Guido Münch (these proceedings) has looked at Halpha from HVCs and he emphasized the great precission required in such measurements because of the faintness of the signal and the sources of contamination.

\section{Interlude on Definitions}

Where does the halo begin, when looking from within the Milky Way disk, and how far does the halo extend? It seems relevant to sort out a few phrases. The early definition of halo referred to the older stars, to lower metallicity, and to spatial extent. In later radio and infrared studies the word halo often has been used to just mean "at high galactic latitudes". That the halo extends far out is, I feel, a notion common among the workers in the field. But where then does the halo start? If we believe that the gas outside the Milky Way disk is largely ionised, then the halo may be thought to start at a $z$ height equal to the scale height of neutral gas.

The velocities need some important comments. High-Velocity originally meant gas at speeds differing by more than $20 \mathrm{~km} \mathbf{s}^{-1}$ from the LSR, a definition based on the reach of optical spectroscopy. Later, with $21-\mathrm{cm}$ measurements of the entire galaxy, the definition of HVC became "cloud with velocity not in agreement with galactic rotation models". This means that in practice the limit in velocity of HVCs is a function of galactic direction: only such "HVCs" are recognizable which do not blend in the normal HI $21-\mathrm{cm}$ emission velocities.

Is high-velocity gas in the halo? It may be but may be it is not. There are numerous examples of high velocity absorption features present in spectra of disk stars. It is likewise possible that high-velocity gas seen at high galactic latitudes is near the disk.

May zero-velocity gas be in the halo? Yes, why not, but one cannot find such gas that easily since it will blend in velocity with any gas present locally in the Milky Way disk.

\section{Distances to the Halo Gas Components}

The location of the base of the halo may, for practical purposes, be taken as the same as the end of the disk. Of course, since gas 
is a diffuse matter there is no borderline between disk and halo so one may use the notion of the scale height of the gas. Lockmann, Hobbs, and Shull (1986) investigated the vertical extent of the neutral gas of the Milky Way. They collected Lyman-alpha data for 40 stars and could for 25 derive reliable HI column densities from the interstellar absorption line. Comparison with HI 21-cm emission in the same directions showed that at most $15 \%$ of the neutral gas is further than $1 \mathrm{kpc}$ from the Milky Way disk. This demonstrates unambiguously the limited thickness of the neutral layer.

The well ionised gas appears to start showing up in absorption lines as of vertical distances of about $1 \mathrm{kpc}$. The investigations of the lines of sight to the Magellanic clouds (Savage and de Boer 1981) showed that the, in those directions normally strong, SiIV and CIV lines (these being proof of well ionised gas) were absent in the spectra of two foreground stars with distances of about 1 kpc. In a subsequent study, Pettini and West (1982) showed that along their 30 sight lines into the halo the absorption by SiIV and CIV started showing up only outside $1 \mathrm{kpc}$, their most distant object being $3 \mathrm{kpc}$ below the Milky Way disk. Related but different is the positive detection of large amounts of SiIV, CIV and $N V$ in the spectra of stars with $z$ about $2 \mathrm{kpc}$ in the direction of the central parts of the Milky Way (Savage and Massa 1987). They detected well ionised gas outside a layer of $1 \mathrm{kpc}$, but the directions probed sample parts of the Milky Way where the disk may be thicker than near the sun. Very accurate data have become available using SN1987A as background light source. This line of sight shows essentially that what was known toward the LMC in general, but in particular the lines from SiIV and CIV, which are very smooth in velocity, show that large amounts of well ionised gas are present in the halo (Savage et al., 1989).

The distribution of free electrons also defines the location of ionised gas in the galaxy and outside the Milky Way disk. Again globular clusters appear to be important in this respect. The newly discovered pulsars in globular clusters allow to add very essential dispersion measures to the data base available. Reynolds (1989) showed that also the electrons may have a spatial distribution with a scale height of 1 to $2.5 \mathrm{kpc}$, very similar to the scale height of the ions SiIV and CIV.

The quest to find distances to HVCs has not ended although in some cases distance limits have been claimed. The number of pertinent studies is rather limited. The first comes from Münch (1952) with 2 cases of absorption at high velocity, but few studies followed. The possibility to observe the dominant ionic stages of the interstellar elements through UV absorption lines, and the detection of two HVCs in front of the LMC (Savage and de Boer, 1979, 1981), increased interest in the halo. Many individual lines of sight were investigated. Pettini and West (1982) observed with the IUE many stars at high latitudes and with $z$-distances up to $3 \mathrm{kpc}$ but found no high velocity absorption. Towards two globular clusters CII was seen in absorption at velocities in the HVC range of up to $-100 \mathrm{~km} \mathrm{~s}^{-1}$ (de Boer and Savage, 1983, 1984). Very nice results are being obtained by Danly (1989) who has analysed IUE spectra of high-latitude stars and she finds in some cases absorption at velocities up to $-90 \mathrm{~km} \mathrm{~s}^{-1}$. In the visual a very 
Table 1. Sight lines with detected high-velocity gas in spectra of objects with $z>0.7 \mathrm{kpc}$ and with gas velocities $>/ 50 / \mathrm{km} \mathrm{s}^{-1}$

\begin{tabular}{|c|c|c|c|c|c|c|c|c|}
\hline Object & 1 & b & type & $d$ & $z$ & $\begin{array}{l}v \text { (rad } \\
\text { abs. }\end{array}$ & 1) $21-\mathrm{cm}$ & ref \\
\hline $\begin{array}{l}\text { M10 } \\
\text { vZ1128 M3 } \\
\text { B29 M13 } \\
\text { HD215733 } \\
\text { BT Dra } \\
\text { HD121800 } \\
\text { HZ 25 } \\
\text { HDE } 233622 \\
\text { BD+38 } 2182 \\
\text { HD } 93521 \\
\text { HD100340 } \\
\text { LMC }\end{array}$ & $\begin{array}{r}15 \\
42 \\
59 \\
85 \\
99 \\
113 \\
153 \\
168 \\
182 \\
183 \\
259 \\
280\end{array}$ & $\begin{array}{l}+23 \\
+79 \\
+41 \\
-36 \\
+51 \\
+50 \\
+80 \\
+44 \\
+62 \\
+62 \\
+61 \\
-33\end{array}$ & $\begin{array}{l}\text { G.C. } \\
\text { G.C. } \\
\text { G.C. } \\
\text { B1II } \\
\text { RR LYr } \\
\text { B1.5V } \\
\text { B2: } \\
\text { B1.5: } \\
\text { B2: } \\
\text { O9V } \\
\text { B1IV } \\
\text { OB* }\end{array}$ & $\begin{array}{c}4.3 \\
10 \\
6.3 \\
2.5 \\
2.1 \\
2.2 \\
3.3: \\
3.5: \\
4.6: \\
2.1 \\
5.7 \\
55 \\
4.3:\end{array}$ & $\begin{array}{c}1.7 \\
10 \\
4.1 \\
-1.5 \\
1.6 \\
1.7 \\
3.3: \\
2.4: \\
4.1: \\
1.8 \\
5.0 \\
30\end{array}$ & $\begin{array}{l}-78 \\
-70 \\
-80 \\
-50 \\
-136 \\
-113 \\
-91 \\
-72 \\
-96 \\
-53 \\
-70 \\
-60 \\
-120 \\
-77\end{array}$ & $\begin{array}{r}-85 \\
-80 \\
-50 \\
-140\end{array}$ & $\begin{array}{l}\text { KK7 } \\
\text { BS84 } \\
\text { BS83 } \\
\text { A83 } \\
\text { SCW88 } \\
\text { D89 } \\
\text { D89 } \\
\text { D89 } \\
\text { D89 } \\
\text { M52, A83 } \\
\text { D89 } \\
\text { SB81 } \\
\text { SB81 } \\
\text { D89 }\end{array}$ \\
\hline
\end{tabular}

0) PW82 quote $\mathrm{d}=0.8 \mathrm{kpc}$ and find no HV absorption in FeII

refs.: A83= Albert 1983 (CaII), BS84= de Boer and Savage 1984 (CII), $B S 83=$ de Boer and Savage 1983 (CII), D89= Danley 1989 (CII), KR72= Kerr and Rnapp 1972 (CaII), M52= Münch 1952 (CaII), PW82= Pettini and West 1982 (FeII), SB81= Savage and de Boer 1981 (FeII), SCW8 8 = Songalla, Cowie and Weaver 1988 (CaII)

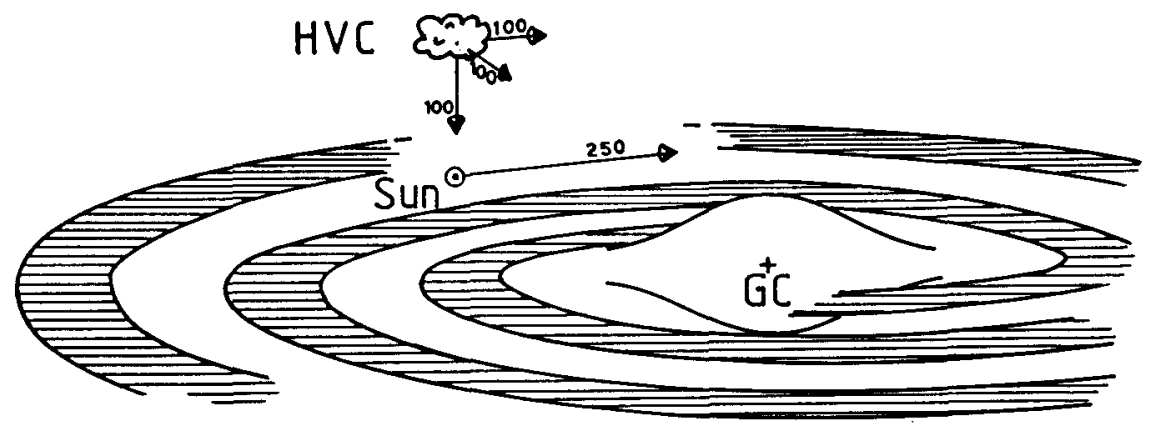

Fig. 1. The observed velocities of almost all HVCs are consistent with the following simplified characteristics: $z$ distances between 2 and $5 \mathrm{kpc}$ and velocity components of the order of $100 \mathrm{~km} \mathrm{~s}-1$ (Kaelble et al 1985), as indicated. Figure from de Boer (1985). 
elegant study was carried out by Albert (1983) who measured TiII, CaII and NaI in pairs of stars with very similar directions but with very different distances. Two cases were found with absorption at velocities differing by more than $40 \mathrm{~km} \mathrm{~s}^{-1}$ from the LSR. This work is being continued by Blades et al (see these proceedings). Reenan et al. (1988) completed a programme on CaII observations of halo stars without finding HV absorption. Songaila, Cowie, and Weaver (1988) published CaII data on stars in the direction of HVgas complexes of the northern hemisphere and they see CaII absorption in spectra of an RR Lyr star at velocities related to those of the Complex $c$. The intensive investigations (star counts and radio lines) of the Draco Cloud (see Rohlfs et al 1989, Mebold, these proceedings) show that a HVC collides with Milky Way gas at a z-height of over $0.5 \mathrm{kpc}$.

From just HI 21-cm measurements (one observes after all only one velocity component with a cloud direction) it is not possible to derive the distance of individual HVCs. However, if one assumes that all high-latitude HVCs belong to one category it may be possible to arrive at rough values for their kinematic behaviour. Using the HVC-survey of Giovanelli (1980) as database and including the limits on cloud motions derived from the UV absorption lines seen in globular clusters stars (de Boer 1982, de Boer and Savage 1983, 1984), a consistency analysis was carried out by Raelble, de Boer and Grewing (1985). They showed that most of the observed velocities fitted to the following description (see Fig.1):

* a velocity of up to $100 \mathrm{~km} \mathrm{~s}^{-1}$ in the direction of the disk,

* a velocity toward the rotation axis of the Milky way of up to $100 \mathrm{~km} \mathrm{~s}^{-1}$,

* a velocity in the sense of the galactic rotation of about $100 \mathrm{~km} \mathrm{~s}^{-1}$,

* z-distances of 2 to $5 \mathrm{kpc}$.

It was noted that forcing the HVC sample to be at a distance of e.g. only $1 \mathrm{kpc}$ from the disk resulted in bizarre dynamic behaviour in the sense that it would require some form of speedy flow (with respect to the LSR) of gas from the first to the third galactic quadrant. Also, the corotation hypothesis for halo-gas, used in most publications as a first order approach to understanding the HVC phenomenon, appeared to be untenable because of resulting very peculiar 3-dimensional behaviour of the HVC sample.

Based on the theory of the galactic fountain (Shapixo and Field, 1976), Bregman (1980) modelled the flow of gas condensing out of the hot and rising part of the fountain. He found that for reasonable condensation locations the pockets of neutral gas would rain down on the Milky Way disk with velocities rather similar to those known for observed HVCs.

All available data giving indications about the distances of HVCs has been collected and positive detections are assembled in Table 1. The selection criteria for inclusion were that the stars had to be at $z>0.7 \mathrm{kpc}$ and the velocities of the gas had to differ by more than $50 \mathrm{~km} \mathrm{~s}^{-1}$ from the LSR. However, many negative results can be found in the literature (see in Albert 1983, de Boer et al 1989, Keenan et al 1988, Münch 1952, Pettini and West 1982, Songaila et al 1988, Danly 1989; also Blades et al, these proceedings), and it turns out that most of these stars are 
not in the direction of known HV gas. The list of negative results is, of course, very inhomogeneous: in the visual one has measured the CaII line region of the spectra, while in the UV mostly the FeII lines were used. This all by itself results in a very different limit in terms of equivalent HI optical depth. Be as it may, HVCs are seen only in spectra of objects at distances larger than 1.5 to $2 \mathrm{kpc}$ from the Milky Way disk, while in objects with zdistances less than $1.5 \mathrm{kpc}$ no $\mathrm{HV}$ gas is found. The observational result agrees with the consistency analysis of the $21-\mathrm{cm}$ HVC sample by Kaelble, de Boer and Grewing (1985) as discussed above.

\section{Observations of the Future}

We have a lot of information on gas in the galactic halo, but only few hard facts. Neutral gas is abundant in the disk and well ionised gas starts showing up outside $z=1 \mathrm{kpc}$. This in fact defines the "disk" and gives the onset of the "halo". In the halo one finds the high-velocity clouds, in general not nearer than $1 \mathrm{kpc}$, although there are a few clear cases suggesting collisions of HVCs with disk material. Ionised gas can be observed but not the hottest component which would have been detectable through ovI.

New and deeper HI $21-\mathrm{cm}$ surveys looking for HI at high galactic latitudes will probably show that there is HV gas everywhere, i.e., in all directions but $21-\mathrm{cm}$ data will not bring the answer of distances any nearer than it is now. Radio-synthesis maps of individual clouds are needed to verify the usefulness of the stellar probes (Wakker, these proceedings).

Absorption line studies are the most promising but one essential problem here is that one needs a background light source of the proper kind. Extragalactic sources are of limited value; they usually are diffuse and provide limited numbers of photons and their distances are irrelevant for the questions related with the location of halo gas. Thus the best objects for absorption-line studies are stars. For studies in the visual the stellar spectrum has to be devoid of structure at the location of the interstellar absorption lines (CaII or possibly NaI). This means that the stars used either must have wide and shallow lines (fast rotators) or hardly any lines at all (hot stars). Both requirements are difficult to meet. The further away in the halo, the older the stars are (assuming no star formation in the halo) and such stars are cool and slow rotators (Petersson et al., 1983 and further papers). That leaves us with hot and old stars, which are of the subdwarf kind, or generally speaking, horizontal-branch like stars. These stars form an effective temperature continuum which runs from $7000 \mathrm{~K}$ for RR LYr stars via HBA, HBB and sdB stars to $10^{\prime} \mathrm{K}$ for sdo stars. There is a difficult trade-off to be made here: the cooler stars are the more luminous ones thus can be seen and used over larger distances but they in general have a complex intrinsic continuum; the hotter stars are less luminous and thus in general are more nearby but they have usually a clean background continuum. This implies that the HBA and HBB stars can be very useful for studies in the visual, while the sdB and sdo stars are rather 
useful for measurements in the UV. In fact, the sdB stars seem to suffer least from the various drawbacks and might be regarded as an optimum choice as background light source. One special characteristic sets RR LYr stars apart: they have shallow lines at their maximum brightness and seem to be useable then, but clearly the moment of maximum brightness must be known.

York et al (1986) have listed the known RR LYr type stars in the direction of known HV complexes and that project eventually resulted in the measurements reported by songaila, Cowie, and Heaver (1988). Also Schwarz and van Woerden and collaborators have used RR LYr stars to look for HVC absorption. Keenan et al (1988) have searched in spectra of normal stars at high latitude; the "normality" remains a decisive factor in the interpretation of their results. Recognizing the value of sdB stars, de Boer, Heber and Richtler (1988) announced their search for sdB stars and a large number of the stars of approximately that kind present in the Palomar-Green catalogue of blue objects (Green et al 1986) have been investigated to verify their nature and to determine the luminosity and thus the distance (Möhler 1988, Möhler, de Boer et al, in preparation).

The technique of recording high dispersion spectra requires as large an amount of photons possible and sensitive detectors. The first requirement can in the visual be met by using big telescopes, but in the UV that is difficult (IUE $45 \mathrm{~cm}$, HST $1 \mathrm{~m}$ ). In the visual, the second requirement will be met by using CCDs with low readout noise and/or photon-counting systems and both of the detector types are available today. The spectral resolution required at all wavelengths is of the order of 1.5104 or equivalent to $20 \mathrm{~km} \mathrm{~s}^{-1}$. UV absorption lines have the highest optical depth available for the detection of neutral gas.

Further H-alpha data on HVCs would be very helpful, even if only upper limits can be obtained. A big improvement here could be achieved by using a bigger telescope (Reynolds works with $15 \mathrm{~cm}$ ). The problem here is the surface-brightness/aperture-field ratio, which can be made substantially larger than the one used by Reynolds when employing focal reducers at larger telescopes.

It is a pleasure to acknowledge the discussions I had with Guido Münch on the history of the early halo gas studies and the organisers of the meeting for their financial support.

\section{Reterences}

Adams, W.S.: 1948, Astrophys. J. 109, 354

Albert, C.A.: 1983, Astrophys. J. 272, 509

Bajaja, E., Cappa de Nicolau, C.E., Cerosime, J.C., Louiseau, N., Martin, M.C., Korras, R., Olano, C.A., Póppel, H.G.L.: 1985, Astrophys. J. Suppl. 58, 143

Bergeron, J., Savage, B.D., Green, R.F.: 1987, in "Exploring the Universe with the IUE Satellite", Ed. Y. Kondo, Reidel, p 703

Blades, J.C., Wheatley, J.M., Panagia, N., Grewing, M., Pettini, M., Mamsteker, W.: 1988, Astrophys. J. 332, L 75

Bregman, J.R.: 1980, Astrophys. J. 236, 577

Danley, L.: 1989, Astrophys. J. in press

de Boer, R.S.: 1982, in IAU Highlights of Astronomy, 6, 657, Reidel

de Boer, K.S.: 1985, Mitt. Astron. Ges. 63, 21 
de Boer, K.S., Savage, B.D.: 1983, Astrophys. J. 265, 210

de Boer, K.S., Savage, B.D.: 1984, Astron. Astrophys. 136, i 7

de Boer, R.S., Heber, U., Richtler, T.: 1988, Astron. Astrophys. 202, 113

de Boer, K.S., Morras, R., Bajaja, E.: 1989, Astron. Astrophys. submitted

de Boer, K.S., Decker, F., Preussner, P.-R., Schwarz, U.J., Tobin, W.. van Noerden, H.: 1989, Astron. Astrophys. submitted

Edgar, R.J., Savage, B.D.: 1989, Astrophys. J. in press

Giovanelli, R.: 1980 , Astron. J. 85,1155

Birth, M., Dahlem, M., Mebold, U., Muller, P.: 1989, Astron. Astrophys. subm.

Hobbs, L.M., Blitz, L., Magnani, L.: 1986, Astrophys. J. 306, L 109

Hulsbosch, A.N.H.: 1985, in "The Kilky Hay Galaxy", IAU Symp. 106, Eds H. van Noerden, R.J. Allen, H.B. Burton; Reidel, p 409

Eulsbosch, A.N.K., Wakker, B.P.: 1988, Astron. Astrophys. Suppl. Ser. 75, 191

Keenan, P.P., Conlon, E.S., Brown, P.J.F., Dufton, P.L.: 1988, Astron. Astrophys. 192, 295

Kerr, F.J., Knapp, G.R.: 1972, Astron. J. 77, 354

Lockmann, F.J., Hobbs, L.M., Shull, J.K.: 1986, Astrophys. J. 301, 380

Low, F.J., et 13 al.: 1984, Astrophys. J. 278, L 19

Magnani, L., de Vries, C.P.: 1986, Astron. Astrophys, 168, 271

Magnani, L., Lada, E.A., Blitz, L.: 1986, Astrophys. J. 301, 395

McGammon, D., Burrows, D.N., Sanders, W.T., Kraushaar, W.L.: 1983, Astrophys. J. 269, 107

HcGee, R.X., Newton, L.M., Morton, D.C.: 1983, Mon. Not. Roy. Astron. Soc. 205, 1191

HcGee, R.X., Newton, L.M.: 1986, Proc. Astron. Soc. Australia 6, 358

Mebold, U., Cernicharo, J., Velden, L., Reif, K., Crezelius, C., Goerigk, W.: 1985, Astron. Astrophys, 151, 427

wohler, S.: 1988, Diploma Thesis Univ. Bonn

Münch, G.: 1952, Pub. Astron. Soc. Pac. 64, 312

Munch, G.: 1957, Astrophys. J. 125, 42

Kunch, G., Zirin, H.: 1961, Astrophys. J. 133, 11

Peterson, R.:, Tarbell, T.D., Carney, B.W.: 1983, Astrophys. J. 265, 972

Pettini, K., Nest, K.A.: 1982, Astrophys. J. 260, 561

Reypolds, R.J.: 1987, Astrophys. J. 323, 553

Reynolds, R.J.: 1989, Astrophys. J. 339, L 29

Rohlfs, R.., Herbstmeier, U., Mebold, U., Winnberg, A.: 1989, Astron. Astrophys. 211,402

Savage, B.D., de Boer, K.S.: 1979, Astrophys. J. 230, L 77

Savage, B.D., de Boer, K.S.: 1981, Astrophys. J. 243, 460

Savage, B.D., Jenkins, E.B., Joseph, C.L., de Boer, K.S.: 1989, Astrophys. J. in press

Savage, B.D., Massa, D.: 1987, Astrophys. J. 314, 380

Shapiro, P.R., Field, G.B.: 1976, Astrophys. J. 205, 762

Songaila, A., Covie, L.L., Heaver, H.: 1988, Astrophys. J. 329, 580

Spitzer, H.: 1956, Astrophys. J. 124, 20

van Hoerden, B., Schwarz, U.J., Hulsbosch, A.N.M.: 1985, in "The Kilky Way Galaxy", IAU Symp. 106, Eds. H. van Woerden, R.J. Allen, W.B. Burton; Reidel, p 387

York, D.G.: 1982, Ann. Rev. Astron. Astrophys. 20, 221

York, D.G., Burks, G.S., Gibney, T.B.: 1986, Astron. J. 91, 354

\section{Discussion:}

VÖLK: How strong is the empirical evidence against corotation already at heights $|Z|=10 \mathrm{kpc}$ ? Theoretically, one should expect corotation certainly up to about $25 \mathrm{kpc}$, because up to this heights the Alfven velocity should exceed systematic fluid speeds of the halo.

de BOER: The absorption line measurements toward M13 (star Barnard e.g.) show most clearly that there is gas which does not rotate along with the disk. M13 is at $1 \sim 60$, $\mathrm{b} \sim 45$ and distance about $6 \mathrm{kpc}$, so $\mathrm{Z}=4 \mathrm{kpc}$. Absorption is seen down to about $-100 \mathrm{kms}^{-1}$ whereas $+20 \mathrm{kms}^{-1}$ is expected with corotation. This means that the gas between us and M13 stays behind, unless it has an extreme peculiar velocity. The analysis of the $21 \mathrm{~cm}$ HVC data base confirms in other directions that these clouds do rotate along but with velocities well below the disk $220 \mathrm{kms}^{-1}$ (see Kaelble, de Boer, Grewing 1985). 